46 Revista Latinoamericana de la Papa 20 (1): 46 - 55

ISSN: $1853-4961$

http://www.papaslatinas.org/ojs/index.php/index/oai

\title{
Rendimiento y calidad de tubérculos de clones de papa en cosecha temprana
}

\section{F. S. Gnocato ${ }^{1}$; D. A. Bisognin ${ }^{2 / *}$; P. Kielse ${ }^{3}$; M. Bandinelli ${ }^{4}$}

Recibido: 04/03/2016

Aceptado: $13 / 04 / 2016$

Accessible en linea: Junio 2016

\section{Resumen}

El objetivo de este estudio fue evaluar el rendimiento y la calidad de los tubérculos de papa en cosecha temprana para identificar clones con madurez precoz. Nueve clones avanzados y tres cultivares testigos fueron cosechados en las condiciones de cultivo subtropical del sur del Brasil. Las cosechas fueran realizadas a los 70 y 90 días después de la siembra en el cultivo de otoño y 70 días en la primavera. Los experimentos se realizaron en un diseño de bloques completos al azar con tres repeticiones de ocho plantas por unidad experimental. Fueron determinados: el rendimiento de tubérculos, contenido de materia seca, azúcares reductores y sacarosa en los tubérculos. Los tubérculos producidos en el otoño alcanzaron similar madurez (contenido de materia seca y sacarosa) a los 20 días después de aquellos que fueron cosechados en la primavera. Ninguno de los clones evaluados combina precocidad con calidad de los tubérculos.

Palabras claves adicionales: Solanum tuberosum, precocidad, sacarosa, materia seca, azúcares reductores.

\section{Yield and tuber quality of potato clones under early harvest}

\section{Summary}

The aim of this work was to evaluate yield and tuber quality of potato tubers in early harvest to identify clones with early maturity. Nine advanced clones and three check cultivars were early harvested in subtropical grown conditions of southern Brazil. Tubers were harvested at 70 and 90 days after planting in the autumn grown season and at 70 days in the spring one. The experiments were in a random block design, with three replications of eight-hill plots. Yield, dry matter, reducing sugars and sucrose contents in the tubers were determined. Tubers produced during autumn got similar maturity (dry matter and sucrose contents) 20 days after than those produced during spring season. Any of the evaluated clones have early harvest combined with tuber quality.

Additional keywords: Solanum tuberosum, earliness, sucrose, dry matter, reduced sugars.

Autor para correspondencia. Correo electrónico: dilson.bisognin@ufsm.br

${ }^{1,4}$ Programa de Post-Grado en Agronomía, Universidad Federal de Santa Maria, Av. Roraima 1000, Camobi, Santa Maria, RS, Brasil.

2 Profesor Asociado del Departamento de Fitotecnia, Universidad Federal de Santa Maria, Av. Roraima 1000, Camobi, Santa Maria, RS, Brasil.

3 Investigadora del Programa Nacional de Post Doctoramiento, Universidad Federal de Santa Maria, Av. Roraima 1000, Camobi, Santa Maria, RS, Brasil. 


\section{Introducción}

La adaptación del cultivo a diversos ambientes y el valor nutritivo hacen de la papa (Solanum tuberosum L.) un producto de consumo mundial. La papa es considerada el tercer alimento más importante de la humanidad (CIP, 2011) y constituye alimento básico de países desarrollados y en desarrollo (Hijmans, 2001). Los tubérculos de papa son comercializados directamente en el mercado o para la industria de procesamiento en forma de papas fritas y pre fritas congeladas y para la industria de deshidratados y bebidas finas (Bisognin $\mathrm{y}$ Lovatto, 2012).

La papa es unos de los cultivos de mayor importancia económica del Brasil y se puede sembrar en todos los meses del año en diferentes regiones productoras. En las condiciones de clima subtropical y altitudes por debajo de los $600 \mathrm{msnm}$ se recomiendan las siembras de enero a marzo y de julio a setiembre. En regiones con altitudes superiores a $600 \mathrm{msnm}$, es recomendada la siembra sólo en los meses de octubre a diciembre (Heldwein et al., 2009). Las diferentes condiciones ambientales y de cultivo asociados a la falta de cultivares mejor adaptados resultan en grandes variaciones de rendimiento entre las diferentes regiones productoras.

Un aspecto importante es el ciclo de madurez de las cultivares y las condiciones para el cultivo, especialmente en las siembras de enero a marzo, que no permiten que los tubérculos completen la madurez antes de que ocurran las heladas. Además, la intensidad de la radiación solar, la temperatura del aire y la disminución del fotoperiodo durante el crecimiento y desarrollo de las plantas en este periodo y sus aumento en las siembras de julio a setiembre, requieren el uso de cultivares menos sensibles al fotoperiodo (Bisognin et al., 2008). Por lo tanto, en condiciones subtropicales de dos cultivos anuales es esencial el uso de cultivares precoces, con corta dormancia de los tubérculos y menos sensibles al fotoperiodo; caracteres que no se encuentran en los cultivares que fueron desarrolladas en otros países.

La fase de maduración se puede caracterizar por la madurez fisiológica de las plantas, cuando los tubérculos de papa adquieren el máximo contenido de materia seca (Bisognin y Streck, 2009), o por la madurez química determinada por los niveles de carbohidratos no estructurales en los tubérculos durante la cosecha. La sacarosa es el principal azúcar asociado con tubérculos maduros (Ross y Davies, 1992) y está relacionada con la regulación de la actividad de síntesis de almidón y de la reducción en el metabolismo del azúcar (Geigenberger, 2003). Para garantizar la calidad del procesamiento industrial, el contenido de materia seca de los tubérculos de papa debe ser mayor que $21 \%$, mientras que los azúcares reductores responsables por el oscurecimiento de las fritas, deben tener concentración de menos de $0,3 \%$ en la materia seca (Vendrusculo y Zorzella, 2002). Los bajos niveles de sacarosa son deseables debido a su contribución en la síntesis de azúcares reductores durante el almacenamiento a frío (Sowokinos, 1978).

Estudios de caracterización del desarrollo de plantas de papa indican que el máximo índice de área foliar y el inicio de la tuberización ocurren a los 70 días después de la siembra, en las mismas condiciones de clima donde fue realizado este estudio. Por tanto, este es el momento de realizar las evaluaciones de rendimiento y calidad de los tubérculos con el objetivo de identificar clones con madurez temprana (Zanon, 2013). Además, los clones que presentan rendimiento y calidad de los tubérculos en dos cultivos anuales son indicativos de su menor sensibilidad al fotoperiodo y de su adaptación a las condiciones subtropicales (Bisognin et al., 2008), lo que resulta en rendimiento y 
calidad de los tubérculos similares cuando los mismos son cosechados.

El objetivo de este estudio fue evaluar el rendimiento y la calidad de los tubérculos de papa en cosecha temprana para identificar clones con madurez precoz.

\section{Materiales y métodos}

Se realizaron dos experimentos de campo (dos cultivos anuales) en la Fundación de Investigación Agrícola del Estado (FEPAGRO), ubicada en el municipio de Julio de Castilhos $\left(29^{\circ} 10^{\prime} \mathrm{S}\right.$ y $53^{\circ} 41^{\prime} \mathrm{O}$ y 480 msnm), Rio Grande do Sul, Brasil. El clima es cfa, clima subtropical meso térmico húmedo, sin sequía, con temperatura promedio del mes más caliente mayor que $22^{\circ} \mathrm{C}$ y el mes más seco con más de $30 \mathrm{~mm}$ de lluvia, según la clasificación de Köppen (Moreno, 1961). Un experimento fue sembrado el 5 de marzo de 2011 y cosechado a los 70 y 90 días después de la siembra (DDS) (cultivo de otoño). Otro experimento fue sembrado el 14 de setiembre de 2011 y cosechado a los 70 días después de de la siembra (cultivo de primavera).

Se evaluaron los clones avanzados SMINIA793101-3, SMINIA00017-6, SMIJ461-1, SMSJB303-06, SMSJB30507, SMSJB308-14, SMSJB310-07, SMSJB347-08, SMSJB353-01 y los cultivares testigos Asterix, Atlantic y Macaca. En cada experimento, los tubérculos semilla fueron tratados con una solución de $30 \mathrm{mg} \mathrm{L}^{-1}$ de ácido giberélico (Benedetti et al., 2005) para romper la dormancia. Los tubérculos semilla fueron almacenados en condiciones controladas (temperatura de $20^{\circ} \mathrm{C}$ y humedad relativa aproximada de 80\%) hasta la brotación completa (Bisognin y Streck, 2009). En el surco de siembra fueron aplicados la cantidad de $2000 \mathrm{~kg} \mathrm{ha}^{-1}$ de fertilizante formulado que contiene $5 \%$ de nitrógeno $(\mathrm{N}), 20 \%$ de fósforo $\left(\mathrm{P}_{2} \mathrm{O}_{5}\right)$ y $10 \%$ de potasio $\left(\mathrm{K}_{2} \mathrm{O}\right)$. A los 40 días DDS se aplicó $50 \mathrm{~kg} \mathrm{ha}^{-1}$ de $\mathrm{N}$ adicional en el aporque.
El diseño experimental fue de bloques completos al azar con tres repeticiones de ocho plantas por unidad experimental. Las plantas fueran separadas a $0,75 \mathrm{~m}$ y $0,30 \mathrm{~m}$ entre los surcos. En la cosecha se determinó el rendimiento de tubérculos por planta y el contenido de materia seca, azúcares reductores y sacarosa. El rendimiento fue calculado dividiendo la producción total de la parcela entre el número de plantas y fue expresado en gramos de tubérculos por planta. Para cada parcela, se recogió una muestra de tubérculos, que fueran cortados en cubos de $1 \mathrm{~cm}$ y la misma divida en dos partes. La primera porción de la muestra se molió en un mezclador para obtener el extracto acuoso que se utilizó para la cuantificación de sacarosa, por una reacción con antrona descrito en Work et al. (1981). Con la segunda porción de la muestra se determinó la materia fresca y luego después se secó en horno de ventilación forzada a $60^{\circ} \mathrm{C}$ hasta peso constante para cuantificar la materia seca. La cuantificación de los azúcares reductores fue hecho en la materia seca, conforme el método 2,4-dinitrofenol propuesto por Long y Chism (2010) con adaptaciones de Freitas et al. (2006). Todas las determinaciones colorimétricas se realizaron en duplicadas.

Los datos de cada cosecha se sometieron a análisis de varianza según el modelo matemático $Y_{i j}=\mu+G_{i}+B_{j}+\varepsilon_{i j}$, siendo $Y_{i j}$ el valor observado para el genotipo i en el bloque j; $\mu$ la media general; $G_{i}$ el efecto del genotipo i-ésimo; $B_{j}$ el efecto del $\mathrm{j}$ ésimo bloque; y $\varepsilon_{i j}$ el error aleatorio. La relación entre el mayor y menor cuadrado medio residual fue inferior a 7 (prueba del F máximo), lo que indica la varianza residual homogénea, que permitió el análisis conjunto de las pruebas (Cruz et al., 2014). Para el análisis conjunto se utilizó el modelo fijo de acuerdo con el modelo matemático $Y_{i j k}=\mu+G_{j}+A_{i}+G A_{i j}+B / A_{j k}+\varepsilon_{i j k}$, siendo $Y_{i j k}$ el valor observado para el 
genotipo i en la repetición $\mathrm{k}$, en el ambiente j; $\mu$ la media general; $G_{i}$ el efecto del i-ésimo genotipo; $A_{j}$ el efecto del ambiente j-ésimo; $G A_{i j}$ el efecto de la interacción del i-ésimo genotipo con el jésimo ambiente; $B / A_{j k}$ el efecto del k-ésimo bloque dentro del j-ésimo ambiente; y $\varepsilon_{i j k}$ el error aleatorio. Los análisis se realizaron con la ayuda de Microsoft Office Excel 2007 y del software SAS (SAS Institute, 1999).

\section{Resultados y discusión}

El efecto del clon fue significativo ( $\mathrm{p} \leq$ $0,05)$ para el rendimiento y la calidad de los tubérculos en las tres cosechas, a excepción del rendimiento a los 70 DDS del cultivo de otoño (Tabla 1). Estos clones avanzados fueron seleccionados previamente para adaptarse a dos cultivos anuales, lo que limita la variabilidad genética para la producción de tubérculos. Las evaluaciones de la calidad de los tubérculos en la cosecha temprana mostraron diferencias entre los mismos. En el análisis combinado de varianza, el efecto de la interacción de los ambientes con clones fue significativa para el rendimiento, los azúcares reducidos y para la sacarosa (Tablas 1 y 2), y el efecto principal del clon $\mathrm{y}$ del ambiente fue significativo para la materia seca (Tablas 1 y 3$)$.

Tabla 1. Resumen de la análisis de variancia y sus parámetros estadísticos para rendimiento y calidad de los tubérculos de clones de papa en cosecha temprana.

\begin{tabular}{|c|c|c|c|c|c|}
\hline Fuente de variación & GL & Rendimiento $^{3}$ & Materia seca & $\begin{array}{c}\text { Azúcares } \\
\text { reductores }\end{array}$ & Sacarosa $^{5}$ \\
\hline 70 DDS otoño & & \multicolumn{4}{|c|}{ Cuadrado medio } \\
\hline Clones $^{1}$ & 11 & 9087,89 & $2,97 * *$ & $146,27 *$ & $66,31 * *$ \\
\hline Bloques & 2 & 4250,81 & 1,58 & 106,48 & $112,49 * *$ \\
\hline Residuo & 22 & 4376,80 & 0,54 & 48,39 & 10,01 \\
\hline Promedio & & 196,99 & 16,80 & 16,72 & 13,30 \\
\hline $\mathrm{CV} \%$ & & 33,58 & 4,38 & 41,59 & 23,78 \\
\hline 90 DDS otoño & & \multicolumn{4}{|c|}{ Cuadrado medio } \\
\hline Clones & 11 & $26349,95^{*}$ & $2,57 *$ & $181,27 * *$ & $59,73 * *$ \\
\hline Bloques & 2 & $58107,15 * *$ & $8,48 * *$ & 86,23 & $81,49 *$ \\
\hline Residuo & 22 & 9450,96 & 0,99 & 46,13 & 14,99 \\
\hline Promedio & & 402,77 & 18,65 & 24,00 & 11,44 \\
\hline $\mathrm{CV} \%$ & & 24,14 & 5,33 & 28,30 & 33,84 \\
\hline 70 DDS primavera & & \multicolumn{4}{|c|}{ Cuadrado medio } \\
\hline Clones & 11 & $14260,54 *$ & $4,57 * *$ & $360,96 * *$ & $13,13 * *$ \\
\hline Bloques & 2 & 7516,66 & 1,38 & 94,69 & 0,72 \\
\hline Residuo & 22 & 5421,52 & 1,37 & 53,86 & 2,63 \\
\hline Promedio & & 237,58 & 19,02 & 44,29 & 9,33 \\
\hline $\mathrm{CV} \%$ & & 30,99 & 6,15 & 16,57 & 17,37 \\
\hline Conjunto & & \multicolumn{4}{|c|}{ Cuadrado medio } \\
\hline Clones & 11 & $26784,66 * *$ & $8,06^{* *}$ & $362,18 * *$ & $99,31 * *$ \\
\hline Ambientes $^{2}$ & 2 & $427664,90 * *$ & $51,02 * *$ & $7345,11 * *$ & $142,33 * *$ \\
\hline Bloques/ Ambiente & 6 & $23291,11 * *$ & $3,81 * *$ & 95,79 & $64,91 * *$ \\
\hline Clone x Ambiente & 22 & $11456,65^{*}$ & 1,02 & $163,13 * *$ & $19,93 * *$ \\
\hline Promedio & & 279,11 & 18,16 & 28,34 & 11,36 \\
\hline $\mathrm{CV}(\%)$ & & 28,7 & 5,41 & 24,82 & 26,72 \\
\hline
\end{tabular}

${ }^{1}$ Clones avanzados y cultivares testigos Asterix, Atlantic e Macaca; ${ }^{2} 70$ y 90 días después de la siembra de otoño y 70 días después de la siembra de primavera; ${ }^{3}$ Materia fresca (g) de tubérculos por planta; ${ }^{4} \mathrm{mg}$ por $\mathrm{g}$ de materia seca; ${ }^{5} \mathrm{mg}$ por $\mathrm{g}$ de materia fresca; $* * \mathrm{y} *$ significativo al $1 \%$ y $5 \%$, respectivamente, por el teste $\mathrm{F}$; DDS, días después de la siembra; GL, grados de libertad. 
Tabla 2. Rendimiento y calidad de los tubérculos de clones de papa en cosecha temprana.

\begin{tabular}{|c|c|c|c|c|c|}
\hline Clone & $\begin{array}{c}70 \text { DDS }^{1} \\
\text { (otoño) }\end{array}$ & $\begin{array}{c}90 \text { DDS } \\
\text { (otoño) }\end{array}$ & $\begin{array}{c}70 \text { DDS } \\
\text { (primavera) }\end{array}$ & & Promedio \\
\hline & \multicolumn{4}{|c|}{ Rendimiento $^{2}$} & \\
\hline SMSJB308-14 & 304,69 a $\mathrm{A}$ & $438,34 \mathrm{ab} \quad \mathrm{A}$ & $363,16 \mathrm{a} \quad A$ & A & 368,73 \\
\hline SMINIA00017-6 & $180,25 \mathrm{a} \quad \mathrm{B}$ & 548,57 a $A$ & $261,71 \mathrm{a} \quad \mathrm{E}$ & B & 330,18 \\
\hline Asterix & $270,63 \mathrm{a} \quad \mathrm{B}$ & $479,03 \mathrm{ab} \quad \mathrm{A}$ & $239,51 \mathrm{a} \quad \mathrm{E}$ & B & 329,72 \\
\hline SMSJB305-7 & 211,69 a $\mathrm{A}$ & $363,75 \mathrm{ab} \quad \mathrm{A}$ & 313,23 a $A$ & A & 296,22 \\
\hline SMSJB353-1 & 230,68 a $B$ & $426,94 \mathrm{ab} \quad \mathrm{A}$ & $221,39 \mathrm{a} \quad \mathrm{E}$ & B & 293,00 \\
\hline SMINIA793101- & 174,19 a $\quad$ B & $473,30 \mathrm{ab} \quad \mathrm{A}$ & $218,11 \mathrm{a} \quad \mathrm{E}$ & B & 288,53 \\
\hline Macaca & 194,87 a $B$ & $409,33 \mathrm{ab} \quad \mathrm{A}$ & $232,60 \mathrm{a}$ & B & 278,93 \\
\hline SMSJB303-6 & 173,46 a $B$ & $515,34 \mathrm{ab} \quad \mathrm{A}$ & $137,07 \mathrm{a} \quad \mathrm{E}$ & B & 275,29 \\
\hline SMIJ461-1 & 222,38 a $B$ & $315,87 \quad b \quad A$ & $287,58 \mathrm{a} \quad \mathrm{E}$ & B & 275,28 \\
\hline SMSJB310-7 & 146,42 a $B$ & $305,95 \quad b \quad A$ & 276,36 a $A$ & $\mathrm{AB}$ & 242,91 \\
\hline SMSJB347-8 & $108,81 \mathrm{a} \quad \mathrm{B}$ & 295,92 b A & 158,15 a $A$ & $\mathrm{AB}$ & 187,63 \\
\hline \multirow[t]{2}{*}{ Atlantic } & 145,87 a $A$ & 260,86 c A & $142,05 \mathrm{a} \quad A$ & A & 182,93 \\
\hline & & Azúcares redu & & & \\
\hline SMSJB305-7 & 6,00 a $A$ & $18,31 \mathrm{abc} A$ & $36,15 \mathrm{abc}$ & $\mathrm{B}$ & 20,15 \\
\hline SMSJB353-1 & $7,07 \mathrm{a} \quad \mathrm{A}$ & $15,81 \mathrm{ab} \quad \mathrm{A}$ & $43,22 \mathrm{abc}$ & $\mathrm{B}$ & 22,03 \\
\hline SMINIA00017-6 & $19,89 \mathrm{ab} A$ & $13,57 \mathrm{a} \quad \mathrm{A}$ & $35,83 \mathrm{abc}$ & $\mathrm{B}$ & 23,10 \\
\hline SMSJB308-14 & 8,12 a $\mathrm{A}$ & $25,12 \mathrm{abc} \quad \mathrm{B}$ & $36,29 \mathrm{abc}$ & $\mathrm{B}$ & 23,18 \\
\hline SMINIA793101- & $18,43 \mathrm{ab} A$ & 25,12 abc A & $29,68 \mathrm{a}$ & $\mathrm{A}$ & 24,41 \\
\hline Atlantic & $23,20 \mathrm{ab} A$ & 22,73 abc $A$ & $34,50 \mathrm{ab}$ & A & 26,81 \\
\hline SMIJ461-1 & $18,79 \mathrm{ab} A$ & $21,80 \mathrm{abc} A$ & $40,04 \mathrm{abc}$ & $\mathrm{B}$ & 26,87 \\
\hline SMSJB303-6 & $17,22 \mathrm{ab} A$ & $19,27 \mathrm{abc} A$ & 49,42 bcd & B & 28,64 \\
\hline Macaca & $15,63 \mathrm{ab} A$ & $36,24 \quad \mathrm{c} \quad \mathrm{B}$ & $49,94 \mathrm{bcd}$ & B & 33,94 \\
\hline SMSJB310-7 & $15,08 \mathrm{ab} A$ & 37,75 c $B$ & $54,14 \mathrm{~cd}$ & $\mathrm{C}$ & 35,66 \\
\hline Asterix & $21,66 \mathrm{ab} A$ & $19,94 \mathrm{abc} A$ & 67,08 & & 36,23 \\
\hline \multirow[t]{2}{*}{ SMSJB347-8 } & 29,62 b A & 32,34 bc $A$ & $55,17 \mathrm{~cd}$ & $\mathrm{~B}$ & 39,04 \\
\hline & \multicolumn{4}{|c|}{ Sacarosa ${ }^{4}$} & \\
\hline SMSJB308-14 & $6,12 \mathrm{a}$ & $5,47 \mathrm{a} \quad \mathrm{A}$ & $5,54 \mathrm{aA}$ & & 5,71 \\
\hline Atlantic & $6,61 \mathrm{a}$ & $6,31 \mathrm{a} \quad \mathrm{A}$ & $6,67 \mathrm{aA}$ & & 6,53 \\
\hline Macaca & $9,45 a b$ & $6,74 \mathrm{a} \quad \mathrm{A}$ & $10,92 \mathrm{aA}$ & & 9,04 \\
\hline SMSJB303-6 & $11,38 \mathrm{abc}$ & $10,47 \mathrm{ab} \quad \mathrm{A}$ & $8,33 \mathrm{aA}$ & & 10,06 \\
\hline SMSJB353-1 & $11,46 \mathrm{abc}$ & $11,43 \mathrm{ab} \quad \mathrm{A}$ & $9,52 \mathrm{aA}$ & & 10,80 \\
\hline SMSJB347-8 & 13,05 abcdA & $12,94 \mathrm{abcA}$ & $7,80 \mathrm{aA}$ & & 11,26 \\
\hline Asterix & 13,90 abcdA & $9,04 \mathrm{ab} \quad \mathrm{A}$ & $11,13 \mathrm{aA}$ & & 11,35 \\
\hline SMSJB310-7 & 13,29 abcdA & $10,52 \mathrm{ab} \quad \mathrm{A}$ & $11,25 \mathrm{aA}$ & & 11,69 \\
\hline SMSJB305-7 & 18,27 & $13,46 \mathrm{abcAB}$ & $7,96 \mathrm{aA}$ & & 13,23 \\
\hline SMINIA00017-6 & $16,15 \mathrm{bcdA}$ & $16,00 \mathrm{bcA}$ & $10,65 \mathrm{aA}$ & & 14,27 \\
\hline SMINIA793101- & 21,10 & 13,74 abcA & $12,53 \mathrm{aA}$ & & 15,79 \\
\hline SMIJ461-1 & 18,86 & 21,18 c $B$ & $9,64 \mathrm{aA}$ & & 16,56 \\
\hline
\end{tabular}


Tabla 3. Porcentaje de materia seca en los tubérculos de clones de para en cosecha temprana.

\begin{tabular}{lc}
\hline Tratamientos & Materia seca \\
\hline Clones & $19,35 \mathrm{a}$ \\
Atlantic & $18,94 \mathrm{ab}$ \\
SMINIA00017-6 & $18,84 \mathrm{abc}$ \\
SMSJB310-7 & $18,83 \mathrm{abc}$ \\
SMSJB308-14 & $18,74 \mathrm{abc}$ \\
SMSJB303-6 & $18,68 \mathrm{abc}$ \\
SMSJB347-8 & $18,36 \mathrm{abcd}$ \\
SMINIA793101-3 & $18,10 \mathrm{abcd}$ \\
SMIJ461-1 & $17,51 \mathrm{bcde}$ \\
Asterix & $17,30 \mathrm{cde}$ \\
Macaca & $17,07 \mathrm{de}$ \\
SMSJB353-1 & 16,18 \\
SMSJB305-7 & \multicolumn{2}{c}{ e } \\
\hline Ambientes & $19,02 \mathrm{a}$ \\
70 después de la siembra (primavera) & $18,65 \mathrm{a}$ \\
90 después de la siembra (otoño) & $16,80 \mathrm{~b}$ \\
70 después de la siembra (otoño) &
\end{tabular}

No hubo diferencias significativas entre clones para la producción de tubérculos a los 70 DDS en los cultivos de otoño y primavera (Tabla 2). Para los de 90 DDS del cultivo de otoño, el mayor rendimiento fue obtenido con el clon SMINIA00017-6, que difirió significativamente de los clones SMIJ461-1, SMSJB310-7, SMSJB347-8 y la cultivar Atlantic. Este aumento del rendimiento no se puede explicar por la precocidad de la producción de tubérculos, ya que los clones evaluados no difieren a los de 70 DDS, pero pueden ocurrir por una mayor eficiencia fotosintética de las plantas o por una mayor distribución de los nutrientes asimilados a los tubérculos en crecimiento.

En condiciones de clima subtropical de altitud (> $900 \mathrm{~m}$ ), fue identificada una relación directa entre el ciclo tardío y el rendimiento de los tubérculos de papa (Rodrigues et al., 2009; Silva et al., 2009) que sugiere que los clones tardíos deban ser seleccionados con el propósito de aumentar el rendimiento. Sin embargo, en condiciones de clima subtropical y baja altitud, en que el fotoperiodo y la temperatura limitan el desarrollo de cultivares de ciclo tardío, clones de maduración temprana deben ser seleccionados por completar el ciclo en condiciones ambientales más favorables. En estas condiciones fueron identificados clones de papa con inicio de tuberización a los 50 DDS, con amplio rango (mayor de 20 días) entre los clones evaluados (Zanon et al., 2013). En este estudio, la cosecha temprana a los 70 DDS de los cultivos de otoño y de primavera no demostró diferencias significativas entre los clones. La baja variación genética observada entre los clones evaluados puede ser explicada por la selección previa para adaptación a dos cultivos anuales. Esta selección eliminó los clones tardíos y aquellos sensibles al fotoperiodo, que resultan en bajo promedio de rendimiento entre los cultivos otoño y primavera.

Niveles más bajos de azúcares reductores se obtuvieron con los clones SMSJB305-7, SMSJB353-1 y SMSJB308-14, que difieren significativamente del peor clon en 
rendimiento (SMSJB347-8) a los de 70 DDS de otoño (Tabla 2). A los de 90 DDS del cultivo de otoño, los clones con azúcares reductores más bajos fueron: SMINIA00017-6 y SMSJB353-1, que difieren significativamente del clon SMSJB310-7 y la cultivar Macaca, mientras que a los de 70 DDS de la primavera, el clon SMINIA793101-3 y la cultivar Atlantic fueron más altos que los clones SMSJB310-7, SMSJB347-8 y la cultivar Asterix. La menor variación entre los clones con el mayor nivel de azúcares reductores (no deseable en un nuevo cultivar) y la variación entre los clones con niveles más bajos sugieren que la selección solamente se debe realizar con el fin de eliminar los peores clones.

El menor contenido de sacarosa fue obtenido con el clone SMSJB308-14 a los 70 DDS del cultivo de primavera, que difirió significativamente de los clones SMIJ461-1, SMINIA00017-6, SMINIA793101-3 y SMSJB305-7 (Tabla 2). A los 90 DDS de otoño, el clone SMSJB308-14 se mantuvo con bajo contenido de sacarosa, que sólo difirió de los clones SMIJ461-1 y SMINIA00017-6. A los 70 DDS del cultivo de primavera no hubo diferencia significativa entre los clones, lo que, posiblemente, indique que los clones evaluados presentan similar comportamiento en la madurez química. Los cultivares testigos no mostraron variación entre las tres cosechas (tres ambientes), incluso al cosechar a los 70 DDS en el cultivo de otoño, por haber ja adquirido madurez química.

La ocurrencia de condiciones contrastantes entre los cultivos de primavera y otoño interfieren en el proceso de selección de clones de papa para calidad de procesamiento industrial. Los mayores valores de sacarosa observados en algunos clones en el cultivo de otoño (Tabla 2) pueden estar asociados con las condiciones ambientales menos favorables para la maduración de las plantas y tubérculos, pues bajas temperaturas y corto fotoperiodo prolongan la senescencia de las plantas (Heldwein et al., 2009). Los mayores niveles de sacarosa en los tubérculos pueden afectar la calidad del procesamiento industrial, lo que contribuye para el acúmulo de azúcares reductores durante el almacenamiento (Sowokinos, 1978) y causa el oscurecimiento del producto durante la fritura. Estos resultados están de acuerdo con los obtenidos por Freitas et al. (2006) y Augustin et al. (2012), que obtuvieran mayor oscurecimiento de las fritas en el cultivo de otoño.

La comparación entre las épocas de siembra es un indicativo del potencial de los clones para sembrar dos cultivos anuales. El rendimiento no fue diferente en los tres ambientes (cosechas) para los clones SMSJB308-14, SMSJB305-7 y el cultivar Atlantic (Tabla 2). El cultivar Atlantic fue el menos productivo en el otoño a los 90 DDS. Para los azúcares reductores, el cultivar Atlantic y el clon SMINIA793101-3 fueron los más estables, sin diferencias entre los ambientes. Hubo poca variación entre los cultivos para el contenido de sacarosa, con una diferencia significativa sólo para los clones SMIJ4611, SMINIA793101-3 y SMSJB305-7, que tuvieran los niveles más bajos en la primavera. Por lo tanto, existe una variación entre los clones avanzados para la madurez química en la cosecha temprana, siendo que niveles inferiores de sacarosa fueron obtenidos a los 70 DDS de otoño.

El alto contenido de materia seca de los tubérculos de papa está relacionado con ciclos largos y cultivares tardíos (Van Eck, 2007). En este estudio, no hubo interacción entre los clones y ambientes, pero la cosecha de primavera a los 70 DDS y la de otoño a los 90 DDS resultaron en un mayor contenido de materia seca (Tabla 3). El hecho de que el contenido de materia seca fue menor de $21 \%$, siendo que este es el límite inferior recomendado por Vendruscolo y Zorzella (2002) para que 
los tubérculos puedan ser comercializados para la industria de procesamiento, muestra que los tubérculos aún no habían alcanzado el nivel máximo de materia seca, que se esperaba en la cosecha temprana. El cultivar Atlantic obtuvo el mayor contenido de materia seca de los tubérculos, que difirió significativamente de los cultivares Asterix y Macaca y de los clones SMSJB305-7 y SMSJB353-1 (Tabla 3). Las condiciones del cultivo de primavera permiten una mayor acumulación de materia seca en los tubérculos y antes que del cultivo de otoño. Esto está de acuerdo con lo observado en las condiciones de fotoperiodo y radiación solar en el cultivo de primavera, porque son más favorables al desarrollo y madurez de los tubérculos (Freitas et al., 2006; Muller et al., 2009; Bisognin et al., 2008). El efecto significativo de los clones y la no existencia de interacción entre clones con ambientes (cosecha a los 70 DDS de la primavera y a los 70 y 90 DDS del otoño) sugieren que el contenido de materia seca de los tubérculos debe ser utilizado para la identificación de clones superiores de papa en cosecha temprana.

El análisis de correlación parcial mostró una asociación negativa $(r=-0,412)$ entre el contenido de materia seca y sacarosa (Tabla 4), confirmando que la madurez resulta en mayor contenido de materia seca en detrimento de la acumulación de asimilados en los tubérculos (Bisognin y Streck, 2009). Por lo tanto, el contenido de sacarosa indica la madurez química de los tubérculos (Ross y Davies, 1992) y puede también ser utilizada para predecir el potencial de procesamiento de clones de papa después de cuatro a seis meses de almacenamiento (Sowokinos, 1978). Se observó que el contenido de sacarosa se debe medir en tubérculos inmaturos (Nelson y Sowokinos, 1983), claramente confirmado por los resultados de este estudio. La única correlación significativa pero negativa fue entre el contenido de materia seca y de sacarosa, que confirma que la madurez química y fisiológica no ocurren de manera simultánea en los tubérculos. 
Tabla 4. Coeficientes de correlación parcial entre caracteres de productividad y calidad de tubérculos de clones de papa en cosecha temprana.

\begin{tabular}{lccc}
\hline & Massa seca & $\begin{array}{c}\text { Azúcares } \\
\text { reductores }\end{array}$ & Sacarosa $^{3}$ \\
\hline Productividad $^{1}$ & 0,115 & $-0,078$ & $-0,228$ \\
Materia seca & & $-0,135$ & $-0,412 *$ \\
Azúcares redutores $^{2}$ & & & 0,072 \\
\hline
\end{tabular}

${ }^{1}$ Materia fresca $(\mathrm{g})$ de tubérculos por planta; ${ }^{2} \mathrm{mg}$ por $\mathrm{g}$ de materia seca; ${ }^{3} \mathrm{mg}$ por $\mathrm{g}$ de materia fresca.

\section{Conclusiones}

Tubérculos producidos en el otoño alcanzaron una similar madurez (contenido de materia seca y sacarosa) a los 20 días después de los tubérculos producidos en la primavera. Ningún clon evaluado presenta producción temprana combinada con calidad de los tubérculos.

\section{Conflictos de Intereses}

Los autores declaran no tener conflictos de intereses con la publicación de este trabajo de investigación.

\section{Referencias citadas}

Augustin, L.; S. Milach; D.A. Bisognin; M. Suzin. 2012. Genotype x environment interaction of agronomic and processing quality traits in potato. Horticultura Brasileira 30(1): 84-90.

Benedetti, M.; D.A. Bisognin; F.B. Segatto; L.C.Costa; M.G. Bandinelli; A. Brackmann. 2005. Quebra de dormência de minitubérculos de batata. Ciência Rural 35(1): 31-38.

Bisognin, D.A.; M.T. Lovatto. 2012. Tecnologias de processamento para maximizar o aproveitamento de tubérculos de batata. Associação Brasileira da Batata, Itapetininga, $40 \mathrm{p}$.

Bisognin, D.A.; D.R. Müller; N.A. Streck; J.L. Andriolo; D. Sausen. 2008. Desenvolvimento e rendimento de clones de batata na primavera e no outono. Pesquisa Agropecuária Brasileira 43(6): 699-705.
Bisognin, D.A.; N.A. Streck. 2009. Desenvolvimento e manejo das plantas para alta produtividade e qualidade da batata. Associação Brasileira da Batata, Itapetininga, $30 \mathrm{p}$.

CIP. Centro Internacional de la Papa. http://www.cipotato.org/potato/history.asp. Consulta: Octubre, 2011.

Cruz, C.D.; P.C.S. Carneiro; A.J. Regazzi. 2014. Modelos Biométricos aplicados ao melhoramento genético. Universidade Federal de Viçosa, Viçosa, 668 p.

Freitas, S.T.; D.A. Bisognin; A.C. Gómez; C.K. Sautter; L.C. da Costa; M.V. Rampelotto. 2006. Qualidade para processamento de clones de batata cultivados durante a primavera e outono no Rio Grande do Sul. Ciência Rural 36(1): 80-85.

Geigenberger, P. 2003. Regulation of sucrose to starch conversion in growing potato tubers. Journal of Experimental Botany 54(382): 457-465.

Heldwein, A.B.; N.A. Streck; D.A. Bisognin. 2009. Batata, em Monteiro, J.E.B.A. (Org.). Agrometeorologia dos cultivos - $\mathrm{O}$ fator meteorológico na produtividade dos principais cultivos anuais e perenes no Brasil, Ministério da Agricultura Pecuária e Abastecimento, Brasília, p. 91-108, 530 p.

Hijmans, R.J. 2001. Global Distribution of the Potato Crop. American Journal of Potato Research, 78: 403-412. 
Long, A.R.; G.M. Chism. 2010. Physical and chemical methods of evaluation foods, http://food.oregonstate.edu/research/test/re ducing.html. Consulta: Enero, 2010.

Moreno, J.A. 1961. Clima do Rio Grande do Sul. Secretaria da Agricultura, Porto Alegre, $42 \mathrm{p}$.

Müller, D.R.; D.A. Bisognin; J.L. Andriolo; G.R. Morin Jr; F.S. Gnocato. 2009. Expressão dos caracteres e seleção de clones de batata nas condições de cultivo de primavera e outono. Ciência Rural 39(5): 1327-1334.

Nelson, D.C.; J.R. Sowokinos. 1983. Yield and relationships among tuber size, sucrose and chip color in six potato cultivars on various harvest dates. American Potato Journal 60(12): 949-958.

Rodrigues, G.B.; C.A.B. Pinto; F.R.G. Benites; D.S. Melo. 2009. Seleção para duração do ciclo vegetativo em batata e relação com a produtividade de tubérculos. Horticultura Brasileira 27(3): 280-285.

Ross, H.A.; H.V. Davies. 1992. Sucrose metabolism in tubers of potato (Solanum tuberosum L.). Plant Physiology 98:287293.

SAS institute Inc. 1999. SAS ® Companion for the OS/390 Environment, Version 9, SAS Institute Inc., Cary, 526 p.
Silva, F.L.; C.A.B.P. Pinto; J.D. Alves; F.R.G. Benites; C.M. Andrade; G.B. Rodrigues; A.L. Lepre; L.L. Bhering. 2009. Caracterização morfofisiológica de clones precoces e tardios de batata visando à adaptação a condições tropicais. Bragantia 68(2): 295-302.

Sowokinos, J.R. 1978. Relationship of harvest sucrose content to the processing quality and storage life of potatoes. American Potato Journal (55): 333-344.

Van Eck, H.J. 2007. Genetics of morphological and tuber traits, in Vreugdenhil, D. Potato biology and biotechnology: advances and perspectives, Elsevier, p. 91-115. 801 p.

Vendruscolo, J.L.S.; C.A. Zorzella. 2002. Processamento de batata (Solanum tuberosum L.): fritura", Embrapa Clima Temperado, Pelotas, $15 \mathrm{p}$.

Work, T.M.; A.S. Kezis; R.H. True. 1981. Factors determining potato chipping quality. Life Sciences and Agriculture Experiment Station, Orono, $28 \mathrm{p}$.

Zanon Jr, A.; N.A. Streck; B. Kräulich; M.R. Silva; D.A. Bisognin. 2013. Desenvolvimento das plantas e produtividade de tubérculos de batata em clima subtropical. Ciência Agronômica 44(4): 858-868. 